\title{
cAMP signalling inhibits p53 acetylation and apoptosis via HDAC and SIRT deacetylases
}

\author{
MARTINE MÜLLER KLOSTER ${ }^{1}$, ELIN HALLAN NADERI ${ }^{1}$, INGVILD HAALAND ${ }^{2}$, \\ BJøRN TORE GJERTSEN ${ }^{2}$, HEIDI KIIL BLOMHOFF ${ }^{1}$ and SOHEIL NADERI ${ }^{1,3}$ \\ ${ }^{1}$ Institute of Basic Medical Sciences, University of Oslo, N-0317 Oslo; ${ }^{2}$ Institute of Medicine, Hematology Section, \\ Haukeland University Hospital, University of Bergen, N-5021 Bergen, Norway
}

Received November 23, 2012; Accepted January 18, 2013

DOI: $10.3892 /$ ijo.2013.1853

\begin{abstract}
Activation of cAMP signalling potently inhibits DNA damage-induced apoptosis in acute lymphoblastic leukemia cells by promoting the turnover of p53 protein. Recently, we showed that the cAMP-induced destabilization of p53 in DNA-damaged cells occurs as a result of enhanced interaction between $\mathrm{p} 53$ and HDM2. In this report, we present results showing that increased levels of cAMP in cells with DNA damage enhances the deacetylation of $\mathrm{p} 53$, an event that facilitates the interaction of p53 with HDM2, thus annulling the stabilizing effect of DNA damage on p53. The combined inhibition of the HDAC and SIRT1 deacetylases abolished the cAMP-mediated deacetylation of $\mathrm{p} 53$, implying that cAMP-mediated deacetylation of p53 is dependent on the activity of these two classes of histone deacetylases. Importantly, diminishing the activity of HDACs and SIRT1 was also found to reverse the inhibitory effect of cAMP on the DNA damage-induced p53 stabilization and apoptosis, suggesting the involvement of the p53 acetylation pathway in the anti-apoptotic effect of cAMP signalling.
\end{abstract}

\section{Introduction}

p53 is the most frequently mutated tumour suppressor gene in cancers. About $50 \%$ of tumours harbour mutations in the p53 gene, and the remainder have disruptions in the p53 pathway (1). p53 is normally a short-lived protein that is maintained at low levels in resting cells by its negative regulator HDM2. Upon DNA damage induced, for instance, by ionizing radiation (IR), p53 transiently stabilizes and accumulates in the nucleus where it acts as a transcription factor regulating multiple genes important for the regulation of cell cycle arrest, senescence

Correspondence to: Dr Soheil Naderi, ${ }^{3}$ Present address: Unit for Cardiac and Cardiovascular Genetics, Department of Medical Genetics, Oslo University Hospital Rikshospitalet, PO Box 4950 Nydalen, N-0424 Oslo, Norway

E-mail: sohnad@ous-hf.no

Key words: cAMP, p53 acetylation, DNA damage, cell death, deacetylation, acute lymphoblastic leukemia cells and apoptosis. The precise mechanisms of p53 activation are not fully understood, but the activation is clearly dependent on post-translational modifications like ubiquitination, phosphorylation and acetylation (2-4). p53 was the first non-histone protein described to be acetylated (5). Upon various types of stress, acetylation of p53 is dramatically induced, indicating the importance of this specific post-translational modification. There is also a direct competition between acetylation and ubiquitination on specific lysine residues in the C-terminal domain of p53. Acetylation of p53 physically blocks the ubiquitin sites preventing ubiquitination by HDM2 and subsequent degradation (6). Acetylation is also important for recruiting $\mathrm{CBP} / \mathrm{p} 300$ and PCAF to promoter regions for activation of p53-targeted genes such as p21, HDM2 and PUMA. Histone acetyl transferases (HATs) CBP/p300, PCAF and TIP60 can all acetylate p53 (5,7,8), while histone deacetylases (HDACs) can remove acetyl moieties from $\varepsilon-\mathrm{N}$-acetylated lysine residues of histones and non-histone proteins such as p53. Six C-terminal residues (K305, K372, K373, K381, K382 and K386) and one DNA binding domain (DBD) residue (K164) are acetylated by $\mathrm{CBP} / \mathrm{p} 300$ (5,7-10), whereas K320 is acetylated by PCAF $(7,8)$. HDAC inhibitors are also reported to induce acetylation in non-histone proteins (5). The HDAC1 inhibitor TSA leads to acetylation of p53 on K373 primarily under conditions in which cells are subjected to IR, whereas the SIRT1 deacetylase is known to induce acetylation of p53 on K382 (8).

cAMP is a second messenger important in multiple physiological and pathological settings (11). This signal transducer is generated by adenylyl cyclases subsequent to stimulation of certain $\mathrm{G}$ protein-coupled receptors (GPCRs). Our lab has previously reported that elevation of cAMP in lymphoid cells leads to arrest in the G1 phase of the cell cycle (12-14), arrest in the $\mathrm{S}$ phase and inhibition of apoptosis by anticancer agents (15). Using B cell precursor acute lymphoblastic leukaemia (BCP-ALL) cells as a model system, we also reported that the inhibitory effect of cAMP on apoptosis is p53-dependent (16), and that cAMP antagonizes the disruption of p53-HDM2 interaction by DNA damage (17). It was therefore of interest to further investigate the mechanism by which cAMP abolishes IR-induced p53 stability and apoptosis in these cells, and in particular, reveal how cAMP signaling promotes the interaction between p53 and HDM2. Because cAMP exerted only a slight inhibitory effect on IR-induced phosphorylation of S15, T18 and 
S20 on p53 (17), we directed our attention towards the effect of cAMP on p53 acetylation. Our results indicate that cAMP, through inhibition of p53 acetylation, attenuates the IR-induced dissociation of p53 from HDM 2 and, thereby, prevents stabilization of $\mathrm{p} 53$ and apoptosis.

\section{Materials and methods}

Reagents and antibodies. Forskolin (F), PGE2, propidium iodide (PI), cycloheximide (CHX) and nicotinamide (NIA) were obtained from Sigma-Aldrich, 8-CPT-cAMP was purchased from Biolog and Trichostatin A (TSA) was obtained from Calbiochem. Antibodies were: total p53 (DO-1, FL-393, Bp53-12), HDM2 (SMP14) and actin (C2) from Santa Cruz Biotechnology; HDM2 (IF2) from Calbiochem; HDM2 (4B2) was a kind gift from Dr A. Levine (Princeton University, NJ); acetyl-p53 (K373) and acetyl-p53 (K382) were from Epitomics and Cell Signaling, respectively.

Cell cultures, radiation treatment and cell death analysis. The BCP-ALL cell line Reh (18) was cultured as previously described (15). For treatment of cells with $\gamma$-radiation, cells were exposed to a $137 \mathrm{Cs}$ source at a dose rate of $4.3 \mathrm{~Gy} / \mathrm{min}$ using a Gammacell irradiator from MSD Nordion. To analyze cell death, cells were incubated with PI $(20 \mu \mathrm{g} / \mathrm{ml})$ at room temperature for 10 min before examination for PI uptake by flow cytometry.

Two-dimensional SDS-PAGE. For two-dimensional (2D) gel electrophoresis, cells were washed twice in saline and lysed in $7 \%$ trichloro-acetic acid (TCA) for 30 min on ice. After homogenization and centrifugation, the precipitated proteins were washed once in 5\% TCA and three times in water-saturated ether to remove salts, each time followed by centrifugation at $13,000 \mathrm{rpm}$ for $20 \mathrm{~min}$ at $4^{\circ} \mathrm{C}$. The protein pellet was resuspended in sample buffer for 2D gel electrophoresis (7 M urea, $2 \mathrm{M}$ thiourea, $100 \mathrm{mM}$ dithiotreitol, $1.5 \%$ ampholyte $3-10,0.5 \%$ ampholyte $5-6$, $4 \%$ CHAPS). The protein concentration was measured by use of the Bradford method (19). Protein sample $(100 \mu \mathrm{g})$ was diluted in rehydration buffer (7 M urea, $2 \mathrm{M}$ thiourea, $100 \mathrm{mM}$ dithiotreitol, 1.5\% ampholyte 3-10, 0.5\% ampholyte 5-6, 4\% CHAPS, Bromophenol blue) to a final volume of $170 \mu \mathrm{l}$. Isoelectric focusing was performed using $7 \mathrm{~cm} \mathrm{pH} \mathrm{3.0-10.0} \mathrm{(Zoom} \mathrm{Strip,}$ Invitrogen Corp., Carlsbad, CA, USA) isoelectric focusing gel strips. Following rehydration of the strips in rehydration buffer, the strips were subjected to the electrophoresis program: $200 \mathrm{~V}$ for $40 \mathrm{~min}, 450 \mathrm{~V}$ for $30 \mathrm{~min}, 750 \mathrm{~V}$ for $30 \mathrm{~min}$ and $1,000 \mathrm{~V}$ for $1 \mathrm{~h}$. Reduction and acetylation of sulfide bindings were performed according to the manufacturer's procedure. Second dimension gel electrophoresis was run for $1 \mathrm{~h}$ at $200 \mathrm{~V}$. Following electrophoresis, proteins were transferred to polyvinylidene fluoride membrane (Amersham Biosciences AB, Uppsala, Sweden) by standard electroblotting. $\mathrm{p} 53$ protein was detected using primary Bp53-12 antibody (Santa Cruz Biotechnology, Santa Cruz, CA, USA) and secondary HRP-conjugated mouse antibody (Jackson ImmunoResearch, West Grove, PA, USA). Visualization of the proteins was performed using the Supersignal West Pico or Femto Chemiluminescent Substrate system (Pierce Biotechnology Inc., Rockford, IL, USA) and Kodak Image Station 2000R (Eastman Kodak Company, Rochester, NY, USA).
Immunoblot analysis and immunoprecipitation. For immunoblot analysis, cells were lysed in radioimmunoprecipitation buffer [RIPA; $50 \mathrm{mM}$ Tris- $\mathrm{HCl}(\mathrm{pH} 7.4), 150 \mathrm{mM} \mathrm{NaCl}$, $1 \%$ NP-40, $50 \mathrm{mM} \mathrm{NaF}, 10 \mathrm{mM} \beta$-glycerophosphate, $0.1 \%$ SDS, $0.5 \%$ EDTA, $1 \mathrm{mM} \mathrm{Na}_{3} \mathrm{VO}_{4}, 0.2 \mathrm{mM}$ PMSF, $10 \mu \mathrm{g} / \mathrm{ml}$ leupeptin, $0.5 \%$ aprotinin). Equal amounts of protein were separated on a $10 \%$ SDS-PAGE. After transfer to a nitrocellulose membrane (GE Healthcare, Amersham, UK), proteins were detected by use of standard immunoblotting procedures.

For immunoprecipitation of HDM2 in complex with p53, cells were lysed in NP-40 lysis buffer [50 mM Tris (pH 7.5), $150 \mathrm{mM} \mathrm{NaCl}, 0.5 \%$ NP- $40,10 \mathrm{mM} \mathrm{NaF}, 1 \mathrm{mM} \mathrm{Na}_{3} \mathrm{VO}_{4}$, $1 \mathrm{mM}$ phenylmethanesulfonyl fluoride, $10 \mathrm{mg} / \mathrm{ml}$ leupeptin and $0.5 \%$ aprotinin]. Lysates containing $600 \mu \mathrm{g}$ of protein were immunoprecipitated with the 533 antibody FL-393 followed by $50 \mu \mathrm{l}$ of a 1:1 slurry of protein G-agarose (Upstate, Temecula, CA, USA). Beads were washed four times in lysis buffer, eluted in boiling 1X SDS buffer, and subjected to immunoblot analysis. For densitometric analysis, blots were analyzed using Genetools analysis (SynGene).

Statistical analysis. In all figures, the histograms represent the mean values, with error bars corresponding to SEM values.

\section{Results}

cAMP inhibits p53 accumulation and isoelectric point shift in $I R$-treated BCP-ALL cells on $2 D-P A G E$. We have previously shown that stimulation of cAMP signalling inhibits DNA damage-induced accumulation of $\mathrm{p} 53$ by facilitating its interaction with HDM2 (17). Because the interaction between p53 and HDM2 is known to be regulated by post-translational modifications of p53, we decided to examine whether cAMP affected the post-translational modifications of p53 in IR-treated cells. To this end, we performed 2D-immunoblotting with anti-p53 antibodies on lysates of Reh cells that were treated with IR in the absence or presence of forskolin. Reh is a BCP-ALL cell line that expresses wt p53 and forskolin is a plant-derived diterpene known to induce intracellular cAMP levels by activating adenylyl cyclase. As shown in Fig. 1, IR not only led to an increase in the abundance of p53, but it also led to accumulation of more acidic forms of p53, indicating that IR leads to modification of $\mathrm{p} 53$ by phosphorylation or acetylation. Interestingly, forskolin was found to inhibit the IR-induced accumulation and acidification of p53, suggesting that cAMP protects p53 from IR-induced post-translational modifications.

cAMP affects the DNA damage-induced acetylation of K373 and $K 382$ on $p 53$. Our recent result showing that cAMP has only a slight inhibitory effect on the IR-mediated phosphorylation of p53 (17), suggested that inhibition of acetylation might account for the ability of cAMP to reduce the IR-induced acidification of p53. To assess this assumption, Reh cells that were exposed to IR in the absence or presence of forskolin were harvested at $4 \mathrm{~h}$ post-IR and subjected to immunoblot analysis with antibodies specific for p53 acetylated at K373 and K382. Acetylation of p53 at these two residues is characteristically induced by IR (4). As shown in Fig. 2, IR led to an increase in acetylation of p53 at K373 and K382, and importantly, activation of the cAMP signalling pathway by forskolin inhibited this acetylation. 

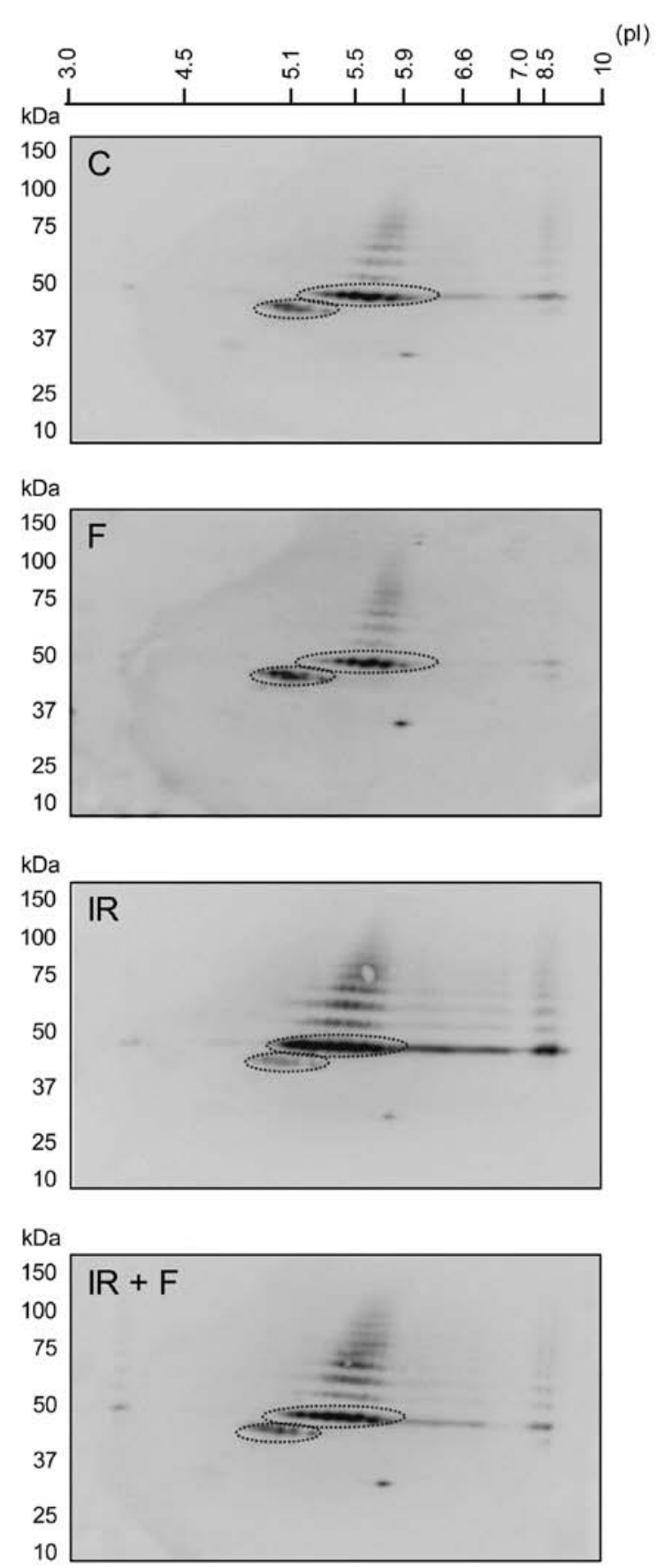

Figure 1. cAMP reverses the accumulation and isoelectric point (pI) of $\mathrm{p} 53$ in IR-treated BCP-ALL cells. Reh cells were pretreated with or without forskolin $(60 \mu \mathrm{M})$ for $30 \mathrm{~min}$ before exposure to IR $(10 \mathrm{~Gy})$. Cells were harvested after $4 \mathrm{~h}$ before they were subjected to 2D-PAGE. The spots inside the large oval shows full length p53 while the spots in the small oval show isoforms of p53.

Following DNA damage, p53 is acetylated through the activity of HATs such as $\mathrm{p} 300 / \mathrm{CBP}$ and pCAF, an event that is tightly regulated by the deacetylase activity of enzymes such as HDACs and SIRT1 (4). The cAMP signalling pathway has been linked to both SIRT activation and subcellular localization of HDACs (20-23), and we therefore hypothesized that cAMP antagonizes the IR-mediated acetylation of p53 through HDACs and/or SIRT1. To test this possibility, we examined the effects of the deacetylase inhibitors trichostatin A (TSA) and
A

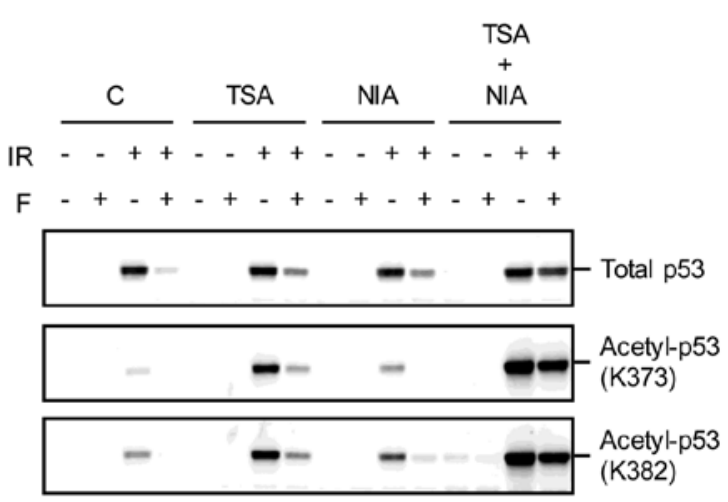

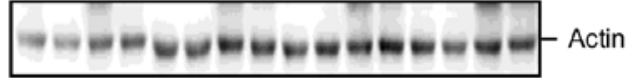

B
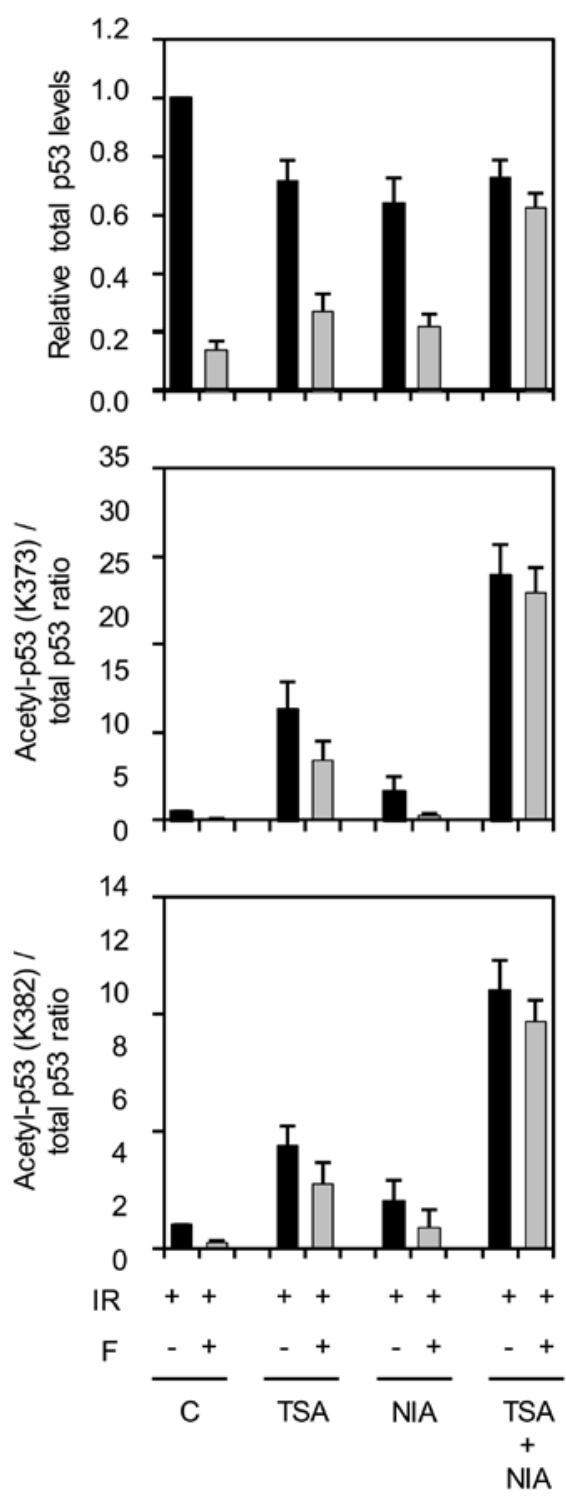

Figure 2. cAMP affects the DNA damage-induced acetylation of K373 and $\mathrm{K} 382$ on p53. (A) Reh cells were preincubated with or without TSA $(0.2 \mu \mathrm{M})$, NIA $(20 \mathrm{mM})$ or a combination of both $2 \mathrm{~h}$ prior to treatment with forskolin $(60 \mu \mathrm{M})$ for $30 \mathrm{~min}$. Cells were then exposed to IR (10 Gy), harvested after $4 \mathrm{~h}$ and subjected to immunoblot analysis with antibodies against total p53 (DO-1), acetylated p53 (K373 and K382) and actin. The immunoblot shows one representative experiment of three. (B) The immunoblots represented in A were subjected to densitometry, the average densitometric values of the p53, K373 and $\mathrm{K} 382$ protein bands as depicted. Treated samples have been normalized to their relevant control whose values were set to 1 . 

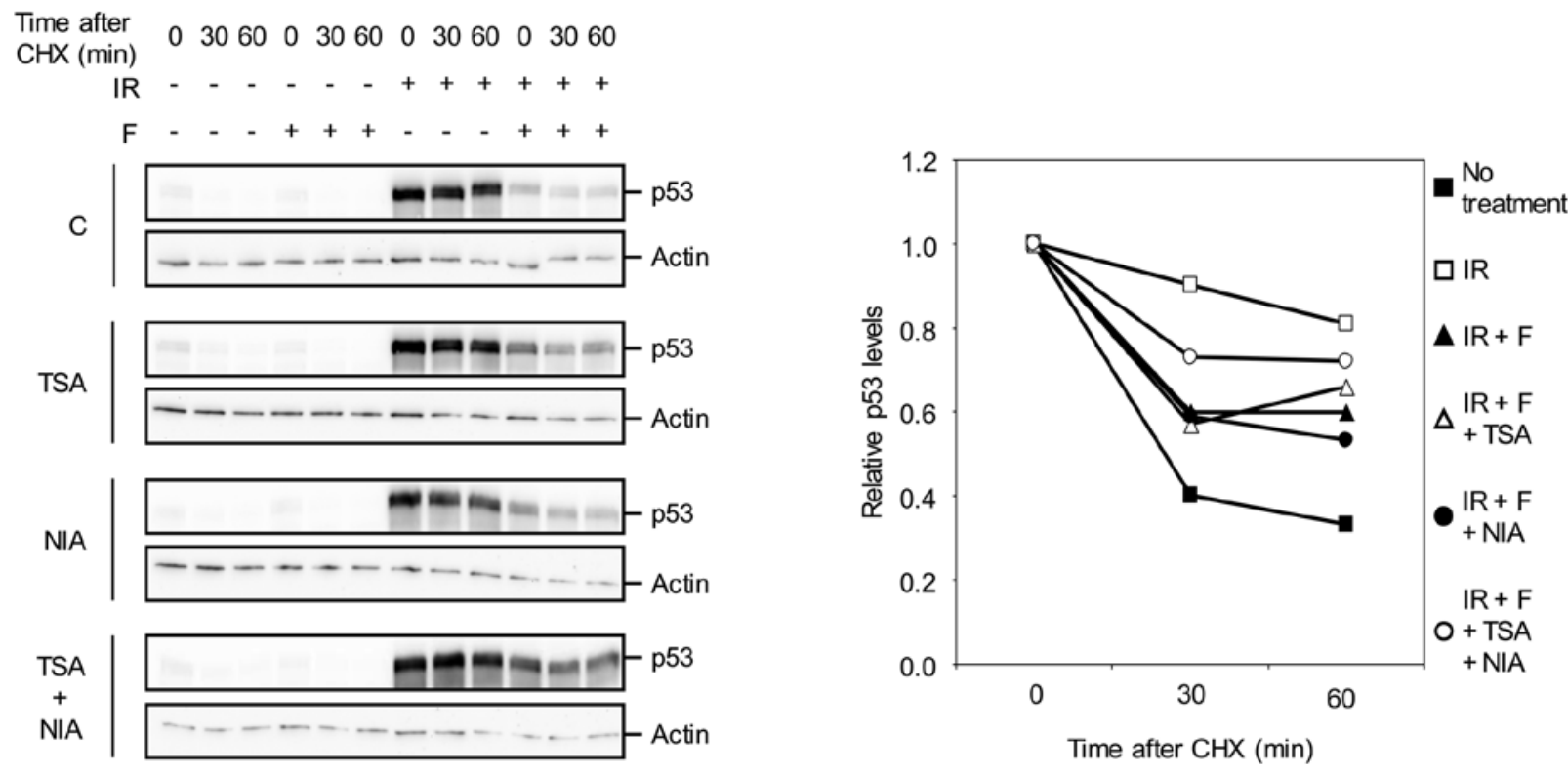

Figure 3. TSA and NIA inhibit the cAMP-induced destabilization of p53 in IR-treated cells. Reh cells were preincubated with or without TSA (0.2 $\mu$ M), NIA $(20 \mathrm{mM})$ or a combination of both $2 \mathrm{~h}$ prior to treatment with forskolin $(60 \mu \mathrm{M})$ for $30 \mathrm{~min}$. Cells were then exposed to IR (10 Gy), and after $4 \mathrm{~h}$ the cells were treated with CHX $(25 \mu \mathrm{g} / \mathrm{ml})$ and harvested at the indicated time points. Whole cell lysates were analyzed by immunoblot analysis using anti-p53 (DO-1) and anti-actin antibodies. The immunoblot shows one reproducible experiment of two. The immunoblots were scanned and the intensity of the p53 protein bands was quantitated and plotted with the value obtained for cells not treated with CHX set as 1.

nicotineamide (NIA) on the ability of cAMP to abrogate the IR-induced acetylation of p53. TSA is an inhibitor of HDACs whereas NIA inhibits the NAD ${ }^{+}$-dependent deacetylases such as SIRT1. To this end, Reh cells were pretreated with TSA and/ or NIA before exposure to IR in the absence or presence of forskolin. Four hours post-IR, cells were harvested and subjected to immunoblot analysis sequentially with antibodies directed against p53 acetylated at K373, K382 as well as total p53. Treatment of cells with TSA or NIA alone or the combination of the two had minor effects on p53 acetylation under unstressed conditions (Fig. 2). In accordance with previous findings (16,24), exposure of cells to IR in the presence of TSA or NIA increased the IR-mediated acetylation of p53. However, pretreatment of cells with both TSA and NIA substantially further enhanced the p53 acetylation induced by IR. Interestingly, Fig. 2A and B also show that pretreatment of IR-exposed cells with both TSA and NIA almost completely abrogated the effect of forskolin on acetylated p53 levels, suggesting that the inhibitory effect of cAMP on IR-induced acetylation of p53 depends on HDACs and SIRT1 activities.

cAMP destabilizes IR-induced p53 through acetylation inhibition. The immunoblot shown in Fig. 2A revealed that in addition to reversal of forskolin-induced deacetylation of $\mathrm{p} 53$, the combination of TSA and NIA also abrogated the inhibitory effect of forskolin on IR-induced accumulation of p53. Given our previous finding that cAMP facilitated the degradation of p53, we wished to examine whether TSA and NIA abolished the inhibitory effect of cAMP on IR-mediated accumulation of p53 by increasing the stability of p53. To this end, Reh cells treated with IR in the absence or presence of forskolin were exposed to the protein synthesis inhibitor cycloheximide (CHX) and then analyzed for the level of p53 by western blot analysis.
In agreement with our previous finding, forskolin substantially decreased the half-life of p53 in IR-treated cells (Fig. 3). Interestingly, combination of TSA and NIA alleviated the inhibitory effect of forskolin on p53 stability in IR-treated cells. This result indicates that simultaneous inhibition of HDACs and SIRT1 antagonizes the destabilizing effect of cAMP on p53, and implicates the modulation of p53 acetylation as the means by which cAMP regulates the stability of p53.

cAMP facilitates p53-HDM2 binding through p53 acetylation. We have shown that cAMP abrogates the DNA damage-induced stabilization of p53 by promoting its interaction with HDM2 (17). This, together with the finding that p53 acetylation has an inhibitory effect on its association with HDM2 $(6,25)$ suggested deacetylation of p53 as the mechanism by which cAMP enhances the p53-HDM2 interaction in IR-treated cells. To assess this hypothesis, we examined the formation of p53-HDM2 complexes under conditions in which the inhibitory effect of cAMP on IR-induced acetylation of p53 is blocked. For this purpose, Reh cells were pretreated with TSA or NIA alone or in combination before exposure to IR in the absence or presence of forskolin. Cells were harvested at $4 \mathrm{~h}$ after IR, and the lysates were subjected to immunoprecipitation with anti-p53 antibodies followed by immunoblot analysis with antibodies against p53 and HDM2. In accordance with our previous results, pretreatment of cells with forskolin prevented the IR-induced dissociation of p53 from HDM2 (Fig. 4). Importantly, TSA and NIA together alleviated the facilitating effect of forskolin on the p53-HDM2 interaction and reduced the level of HDM2 in complex with p53 to a level comparable to that found in IR-only treated cells. This result suggests that cAMP counteracts the IR-induced p53-HDM2 dissociation through inhibition of p53 acetylation. 

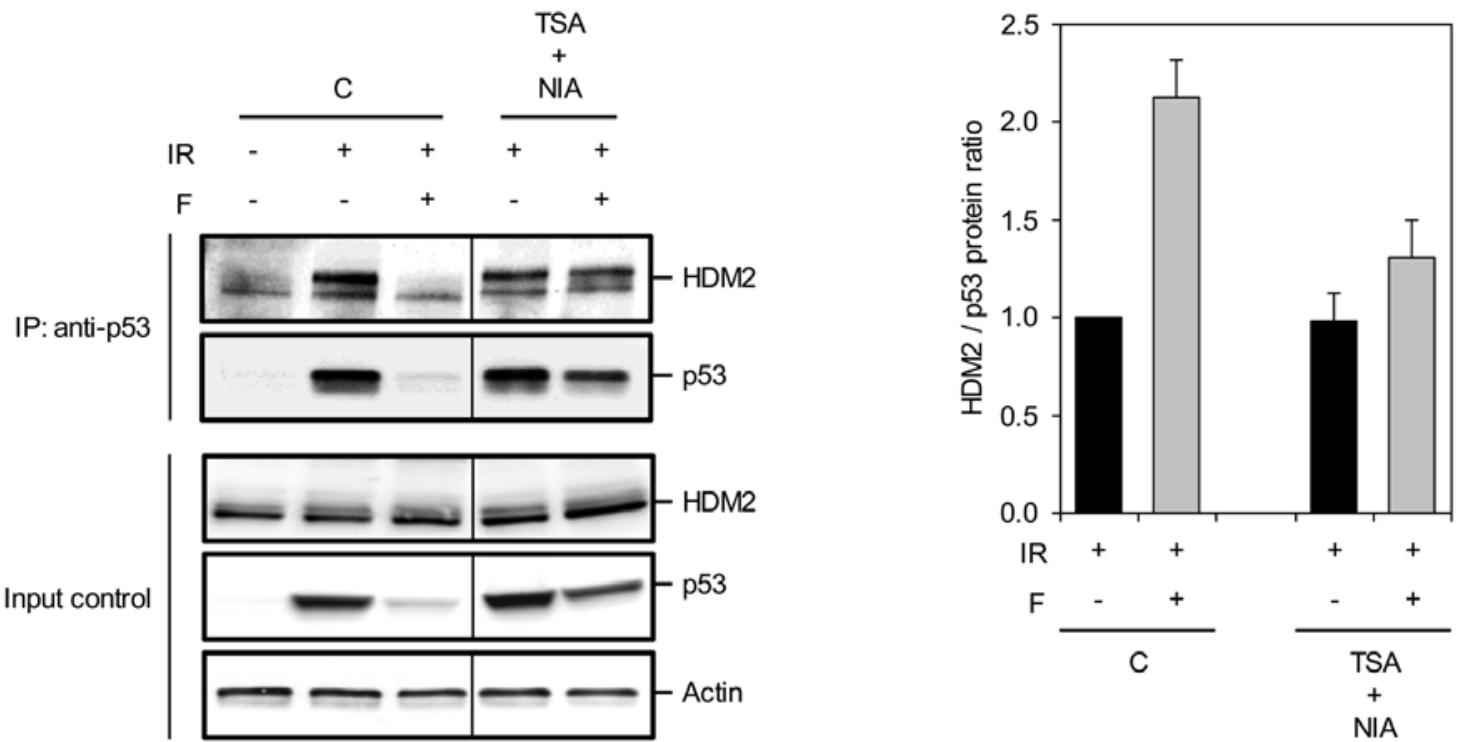

Figure 4. TSA and NIA inhibit the facilitating effect of cAMP on the interaction of p53 with HDM2 in IR-treated cells. Reh cells were pretreated with or without the combination of TSA $(0.2 \mu \mathrm{M})$ and NIA $(20 \mathrm{mM})$ for $2 \mathrm{~h}$ before addition of forskolin $(60 \mu \mathrm{M})$. After $30 \mathrm{~min}$, the cells were exposed to IR (10 Gy) and harvested after $4 \mathrm{~h}$. Whole cell extracts were prepared and subjected to immunoprecipitation with anti-p53 (FL-393). The recovered proteins were resolved on SDS-PAGE and subjected to immunoblot analysis using anti-HDM2 (a combination of SMP14, IF2 and 4B2) and anti-p53 (DO-1) antibodies. The immunoblot in the left panel shows one representative experiment of three. In the right panel, the intensity of protein bands was quantified densitometrically and the ratio of HDM2 relative to p53 was calculated and plotted with the value obtained for cells not treated with forskolin, TSA and NIA set as $1(n=3)$.
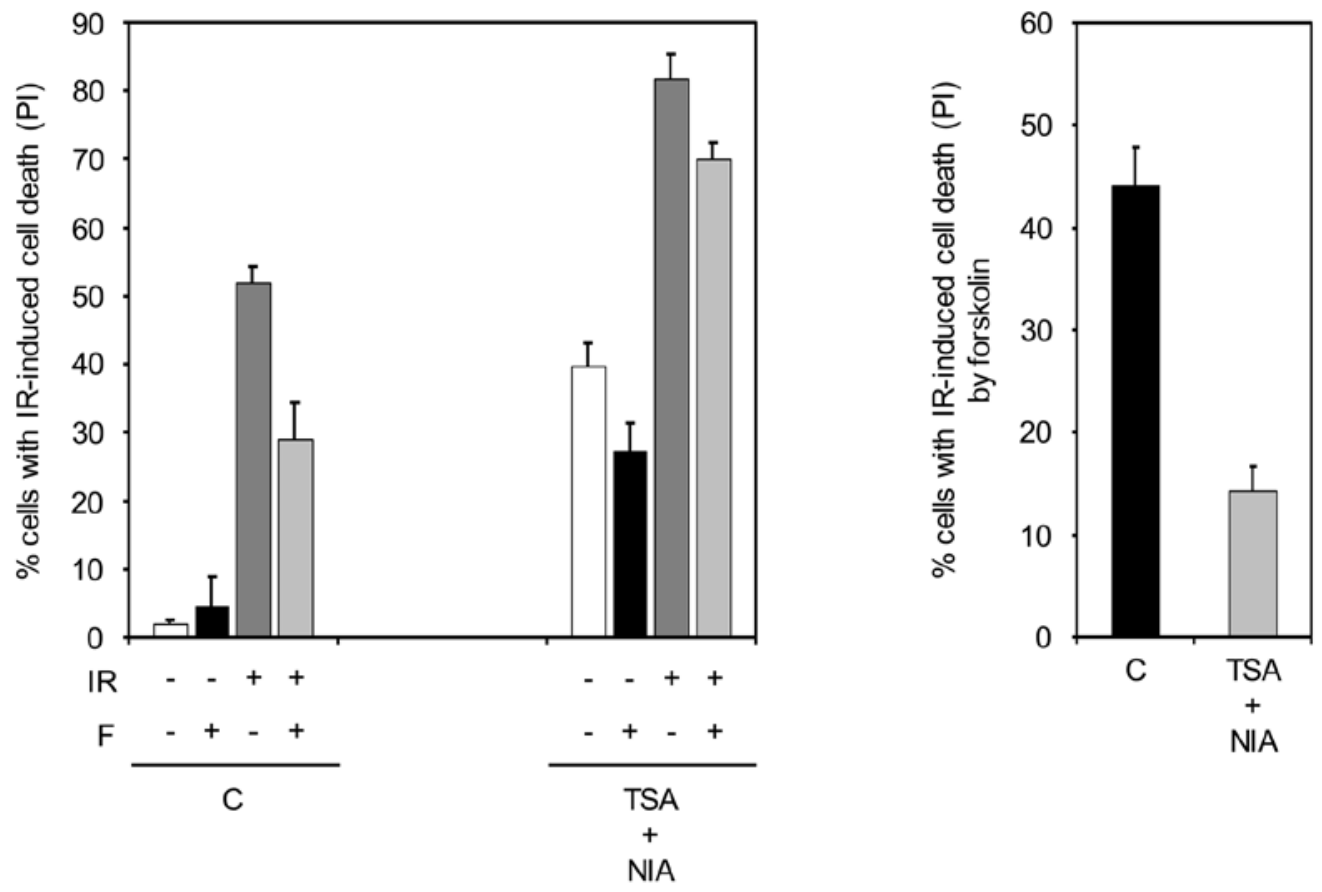

Figure 5. Inhibition of deacetylases attenuates the inhibitory effect of cAMP on IR-induced cell death. Reh cells were pretreated with or without the combination of TSA $(0.2 \mu \mathrm{M})$ and NIA $(20 \mathrm{mM})$ for $2 \mathrm{~h}$ before addition of forskolin $(60 \mu \mathrm{M})$. Cells were then incubated for $20 \mathrm{~h}$ before PI staining and FACS analysis (n=4). The histogram in the right panel depicts percent inhibition of IR-induced cell death by forskolin in the absence or presence of TSA and NIA.

cAMP attenuates the IR-induced apoptosis by inhibiting p53 acetylation. Initiation of a p53-dependent apoptotic program is dependent on acetylation of p53 (10). Given that inhibition of HDACs and SIRT1 abrogates the ability of cAMP to suppress the IR-induced acetylation of p53, we wished to examine whether blocking the HDACs and SIRT1 activities could alle- viate the inhibitory effect of cAMP on IR-mediated apoptosis. To this end, Reh cells were pretreated with TSA and NIA before exposure to IR in the absence or presence of forskolin and then examined for cell death. As shown in Fig. 5, the combined inhibition of HDACs and SIRT1 opposed the inhibiting effect of forskolin on IR-induced cell death, suggesting that inhibition of 
p53 acetylation plays an important role in the ability of cAMP to inhibit IR-mediated cell death.

\section{Discussion}

Elevation of intracellular cAMP levels has an inhibitory effect on DNA damage-induced apoptosis in normal lymphoid cells as well as BCP-ALL cells (16). We have previously shown that this inhibitory effect of cAMP is mediated through its ability to abrogate the DNA damage-induced p53-HDM2 dissociation, leading to restoration of 553 degradation in DNA-damaged cells (17). Here, we provide evidence for the mechanism by which cAMP promotes the interaction of p53 with HDM 2 and thereby prevents DNA damage-induced cell death. We show that activation of cAMP signalling attenuates the acetylation of p53 after DNA damage, thus facilitating the p53-HDM2 association.

The p53 levels are tightly regulated by its HDM2-mediated ubiquitination and the ensuing proteasomal degradation. Because ubiquitnation and acetylation of p53 occur at the same lysine residues, the acetylation of p53 at lysine residues in its C-terminus inhibits the HDM2-mediated ubiquitination and degradation of p53 $(6,26)$. Furthermore, p53 acetylation has also been shown to block its interaction with HDM2 (10). Thus, acetylation of $\mathrm{p} 53$ plays a central role in regulation of $\mathrm{p} 53$ stability and activity following DNA damage by modulating its interaction with HDM2. Acetylation of $\mathrm{p} 53$ is carried out by a number of acetyl transferases, among which p300/CBP is responsible for acetylating the $\mathrm{C}$-terminal lysine residues of p53 (5,7-9). The steady-state level of acetylated p53 is achieved by HDAC1 and SIRT1 deacetylases. Indeed, deacetylation of p53 by these two enzymes is required for restraining hyperactivation of p53 after DNA damage (4). Our finding that cAMP inhibits DNA damage-induced acetylation of $\mathrm{p} 53$ provides a mechanistic explanation for the ability of cAMP to abrogate a p53-dependent response following DNA damage. By inducing the deacetylation of p53, cAMP favours p53-HDM2 interaction and thus abrogates the DNA damage-mediated stabilization of p53. This ability of cAMP requires deacetylation of p53 at both HDAC- and SIRT1-targeted sites, because only simultaneous inhibition of both HDACs and SIRT1 reverses the destabilizing effect of cAMP on p53. In addition, through induction of p53 deacetylation, cAMP attenuates DNA damage-induced events downstream of $\mathrm{p} 53$. This conclusion is supported by the observation that inhibition of HDACs and SIRT1 reverses the inhibitory effect of cAMP on the DNA damage-mediated apoptosis. Thus, cAMP, through modulation of p53 acetylation, inhibits stabilization of p53 and prevents subsequent cell death induced by DNA damage.

In theory, cAMP could inhibit DNA damage-induced acetylation of p53 by two distinct mechanisms: (i) by inhibiting the enzymes that acetylate $\mathrm{p} 53$ or (ii) by stimulating the deacetylases that target $\mathrm{p} 53$. Supported by studies showing that cAMP signalling stimulates the deacetylase activity of SIRT1 and promotes the nuclear retention of HDACs (20-23), we favour the second possibility and suggest that cAMP, by utilizing HDACs and SIRT1, maintains p53 in a hypoacetylated state, thus leading to its HDM2-dependent degradation even in the face of DNA damage. Given the cytotoxic effect of histone deacetylase inhibition $(27,28)$, we are tempted to suggest that combination of inhibitors of cAMP signalling with histone deacetylase inhibitors might prove beneficial for increasing the antitumour activity of histone deacetylase inhibitors.

\section{Acknowledgements}

We thank the University of Oslo, the Norwegian Cancer Society, Anders Jahre's Research Foundation and the Blix Family Foundation for generously supporting this study.

\section{References}

1. Dai $\mathrm{C}$ and $\mathrm{Gu} \mathrm{W}: \mathrm{p} 53$ post-translational modification: deregulated in tumorigenesis. Trends Mol Med 16: 528-536, 2010.

2. Appella E and Anderson CW: Post-translational modifications and activation of p53 by genotoxic stresses. Eur J Biochem 268: 2764-2772, 2001

3. Brooks CL and Gu W: Ubiquitination, phosphorylation and acetylation: the molecular basis for p53 regulation. Curr Opin Cell Biol 15: 164-171, 2003.

4. Brooks $\mathrm{CL}$ and $\mathrm{Gu}$ W: The impact of acetylation and deacetylation on the p53 pathway. Protein Cell 2: 456-462, 2011.

5. Gu W and Roeder RG: Activation of $\mathrm{p} 53$ sequence-specific DNA binding by acetylation of the p53 C-terminal domain. Cell 90: 595-606, 1997.

6. Li M, Luo J, Brooks CL and Gu W: Acetylation of p53 inhibits its ubiquitination by Mdm2. J Biol Chem 277: 50607-50611, 2002.

7. Liu L, Scolnick DM, Trievel RC, Zhang HB, Marmorstein R, Halazonetis TD and Berger SL: p53 sites acetylated in vitro by PCAF and p300 are acetylated in vivo in response to DNA damage. Mol Cell Biol 19: 1202-1209, 1999.

8. Sakaguchi K, Herrera JE, Saito S, Miki T, Bustin M, Vassilev A, Anderson CW and Appella E: DNA damage activates p53 through a phosphorylation-acetylation cascade. Genes Dev 12: 2831-2841, 1998.

9. Wang YH, Tsay YG, Tan BC, Lo WY and Lee SC: Identification and characterization of a novel p300-mediated p53 acetylation site, lysine 305. J Biol Chem 278: 25568-25576, 2003.

10. Tang Y, Zhao W, Chen Y, Zhao Y and Gu W: Acetylation is indispensable for p53 activation. Cell 133: 612-626, 2008.

11. Torgersen KM, Vang T, Abrahamsen H, Yaqub S and Tasken K: Molecular mechanisms for protein kinase A-mediated modulation of immune function. Cell Signal 14: 1-9, 2002.

12. Blomhoff HK, Blomhoff R, Stokke T, deLange DC, Brevik K, Smeland EB, Funderud S and Godal T: cAMP-mediated growth inhibition of a B-lymphoid precursor cell line Reh is associated with an early transient delay in G2/M, followed by an accumulation of cells in G1. J Cell Physiol 137: 583-587, 1988.

13. Blomhoff HK, Smeland EB, Beiske K, Blomhoff R, Ruud E, Bjoro T, Pfeifer-Ohlsson S, Watt R, Funderud S and Godal T: Cyclic AMP-mediated suppression of normal and neoplastic $\mathrm{B}$ cell proliferation is associated with regulation of myc and Ha-ras protooncogenes. J Cell Physiol 131: 426-433, 1987.

14. Naderi S, Gutzkow KB, Christoffersen J, Smeland EB and Blomhoff HK: cAMP-mediated growth inhibition of lymphoid cells in G1: rapid down-regulation of cyclin D3 at the level of translation. Eur J Immunol 30: 1757-1768, 2000.

15. Naderi S, Wang JY, Chen TT, Gutzkow KB and Blomhoff HK: cAMP-mediated inhibition of DNA replication and S phase progression: involvement of Rb, p21Cipl, and PCNA. Mol Biol Cell 16: 1527-1542, 2005.

16. Naderi EH, Findley HW, Ruud E, Blomhoff HK and Naderi S: Activation of cAMP signaling inhibits DNA damage-induced apoptosis in BCP-ALL cells through abrogation of p53 accumulation. Blood 114: 608-618, 2009.

17. Naderi EH, Jochemsen AG, Blomhoff HK and Naderi S: Activation of cAMP signaling interferes with stress-induced p53 accumulation in ALL-derived cells by promoting the interaction between p53 and HDM2. Neoplasia 13: 653-663, 2011.

18. Rosenfeld C, Goutner A, Choquet C, Venuat AM, Kayibanda B, Pico JL and Greaves MF: Phenotypic characterisation of a unique non-T, non-B acute lymphoblastic leukaemia cell line. Nature 267: 841-843, 1977.

19. Bradford MM: A rapid and sensitive method for the quantitation of microgram quantities of protein utilizing the principle of protein-dye binding. Anal Biochem 72: 248-254, 1976. 
20. Cai R, Kwon P, Yan-Neale Y, Sambuccetti L, Fischer D and Cohen D: Mammalian histone deacetylase 1 protein is posttranslationally modified by phosphorylation. Biochem Biophys Res Commun 283: 445-453, 2001.

21. Du M, Perry RL, Nowacki NB, Gordon JW, Salma J, Zhao J, Aziz A, Chan J, Siu KW and McDermott JC: Protein kinase A represses skeletal myogenesis by targeting myocyte enhancer factor 2D. Mol Cell Biol 28: 2952-2970, 2008.

22. Gerhart-Hines Z, Dominy JE Jr, Blattler SM, Jedrychowski MP, Banks AS, Lim JH, Chim H, Gygi SP and Puigserver P: The cAMP/PKA pathway rapidly activates SIRT1 to promote fatty acid oxidation independently of changes in NAD(+). Mol Cell 44: 851-863, 2011.

23. Ha CH, Kim JY, Zhao J, Wang W, Jhun BS, Wong $\mathrm{C}$ and Jin ZG: PKA phosphorylates histone deacetylase 5 and prevents its nuclear export, leading to the inhibition of gene transcription and cardiomyocyte hypertrophy. Proc Natl Acad Sci USA 107: $15467-15472,2010$.
24. Luo J, Nikolaev AY, Imai S, Chen D, Su F, Shiloh A, Guarente L and $\mathrm{Gu}$ W: Negative control of p53 by Sir2alpha promotes cell survival under stress. Cell 107: 137-148, 2001.

25. Wang X, Taplick J, Geva N and Oren M: Inhibition of p53 degradation by Mdm2 acetylation. FEBS Lett 561: 195-201, 2004.

26. Li M, Chen D, Shiloh A, Luo J, Nikolaev AY, Qin J and Gu W: Deubiquitination of $\mathrm{p} 53$ by HAUSP is an important pathway for p53 stabilization. Nature 416: 648-653, 2002.

27. Batty N, Malouf GG and Issa JP: Histone deacetylase inhibitors as anti-neoplastic agents. Cancer Lett 280: 192-200, 2009.

28. Khan O and La Thangue NB: HDAC inhibitors in cancer biology: emerging mechanisms and clinical applications. Immunol Cell Biol 90: 85-94, 2012. 